\title{
Origin of coherent structures in a discrete chaotic medium
}

\author{
Mikhail I. Rabinovich, ${ }^{1}$ Joaquín J. Torres, ${ }^{1}$ Pablo Varona, ${ }^{1,2}$ Ramón Huerta, ${ }^{1,2}$ and Patrick Weidman ${ }^{3}$ \\ ${ }^{1}$ Institute for Nonlinear Science, University of California, San Diego, La Jolla, California 92093-0402 \\ ${ }^{2}$ GNB, ETS Ingeniería Informática, Universidad Autónoma de Madrid, 28049 Madrid, Spain \\ ${ }^{3}$ Department of Mechanical Engineering, University of Colorado, Boulder, Colorado 80309
}

(Received 26 October 1998; revised manuscript received 11 February 1999)

\begin{abstract}
Using as an example a large lattice of locally interacting Hindmarsh-Rose chaotic neurons, we disclose the origin of ordered structures in a discrete nonequilibrium medium with fast and slow chaotic oscillations. The origin of the ordering mechanism is related to the appearance of a periodic average dynamics in the group of chaotic neurons whose individual slow activity is significantly synchronized by the group mean field. Introducing the concept of a "coarse grain" as a cluster of neuron elements with periodic averaged behavior allows consideration of the dynamics of a medium composed of these clusters. A study of this medium reveals spatially ordered patterns in the periodic and slow dynamics of the coarse grains that are controlled by the average intensity of the fast chaotic pulsation. [S1063-651X(99)50708-3]
\end{abstract}

PACS number(s): 05.45. $-\mathrm{a}, 47.54 .+\mathrm{r}$, 95.10.Fh, 84.35.+i

The emergence of spatiotemporal ordered or coherent structures in nonequilibrium media, or in large assemblies of elements with nonregular behavior, is one of the oldest and most intriguing problems in physics, chemistry, and biology. Perhaps the most lucid formulation of the problem is that outlined by fluid dynamicists to explain the observation of large-scale structures in turbulent flows [1]. Over the last decade, neurobiologists have become involved in the problem, particularly after the discovery of spiral patterns in the cortex of animals and humans $[2,3]$.

In this paper we are concerned with regular structures generated by nonregular elements in a diffusive medium characterized by two distinct time (not space) scales. The origin of the ordering phenomena in different nonequilibrium media (or systems) may have some universal features, despite the inherent diversity of specific mechanisms responsible for the formation of the patterns. As many examples indicate, the key feature of this universality is the existence of two or more time or/and space scales in the activity of the media [4-7]. It is therefore reasonable to investigate this ordering phenomenon using a simple, tested model of a twotime-scale nonequilibrium medium. We report here the theoretical and numerical study of a specific model that discloses a possible mechanism responsible for generating such coherent structures.

Let us consider a lattice of $N$ different chaotic generators electrically coupled to their nearest neighbors. Since we wish to investigate the cooperative behavior of an assembly of chaotic generators with fast and slow oscillations, a convenient element of the "medium"' is the Hindmarsh-Rose (HR) [8-10] model neuron. A two-dimensional (2D) lattice composed of such HR elements may be described by the equations

$$
\begin{gathered}
\frac{d x_{i}}{d t}=y_{i}+a x_{i}^{2}-x_{i}^{3}-z_{i}+e_{i}-g \sum_{j}\left(x_{i}-x_{j}\right), \\
\frac{d y_{i}}{d t}=b-c x_{i}^{2}-y_{i},
\end{gathered}
$$

$$
\frac{1}{\mu} \frac{d z_{i}}{d t}=-z_{i}+s\left(x_{i}+d\right),
$$

where the index $j$ runs over the four nearest neighbors of unit $i$, the constants $a, b, c, d, s, e_{i}$, and $\mu$ are model parameters $(\mu \ll 1$ being responsible for the existence of the slow dynamics), and $g$ is the homogeneous coupling strength among neighbor units. Computer simulations of twodimensional square lattices built up with heterogeneous elements (each unit has a random value for the parameter $e_{i}$ ) such as those described by Eqs. (1)-(3) indicate that cooperative behavior among the elements is able to produce large-scale coherent structures with slow periodic oscillations, despite the presence of different and chaotic elements [7]. We define $S$ as the number of neurons that build up the characteristic spatial shape of the structure, and the structure is considered to be of large scale when $S \gg 1$.

In order to understand the origin of these large-scale coherent structures, we investigated the cooperative behavior of a group (cluster) of such chaotic elements. We found a striking phenomenon: the regularization of the average activity when the size of this cluster is sufficiently large. In contrast, small groups of neurons clearly exhibit three different kinds of chaotic dynamics depending on the value of the diffusive coupling $g$ : (i) developed chaos whose dimension increases with the number of chaotic elements for a small value of the coupling, (ii) chaotic synchronization of the slow oscillation (bursts) for moderate coupling, and (iii) complete chaotic synchronization (both spikes and bursts) for strong coupling $[11,12]$.

The cluster with average periodic (in time) behavior will be called a coarse grain. We suppose that the regular spatiotemporal patterns observed in our computer simulations are strongly related to the emergence of many interacting coarse grains inside the lattice for a moderate value of the coupling. The cooperative behavior of the diffusively coupled coarse grains (periodic generators in our case) produce many different regular spatiotemporal patterns similar to those obtained with the discrete analog of the complex 

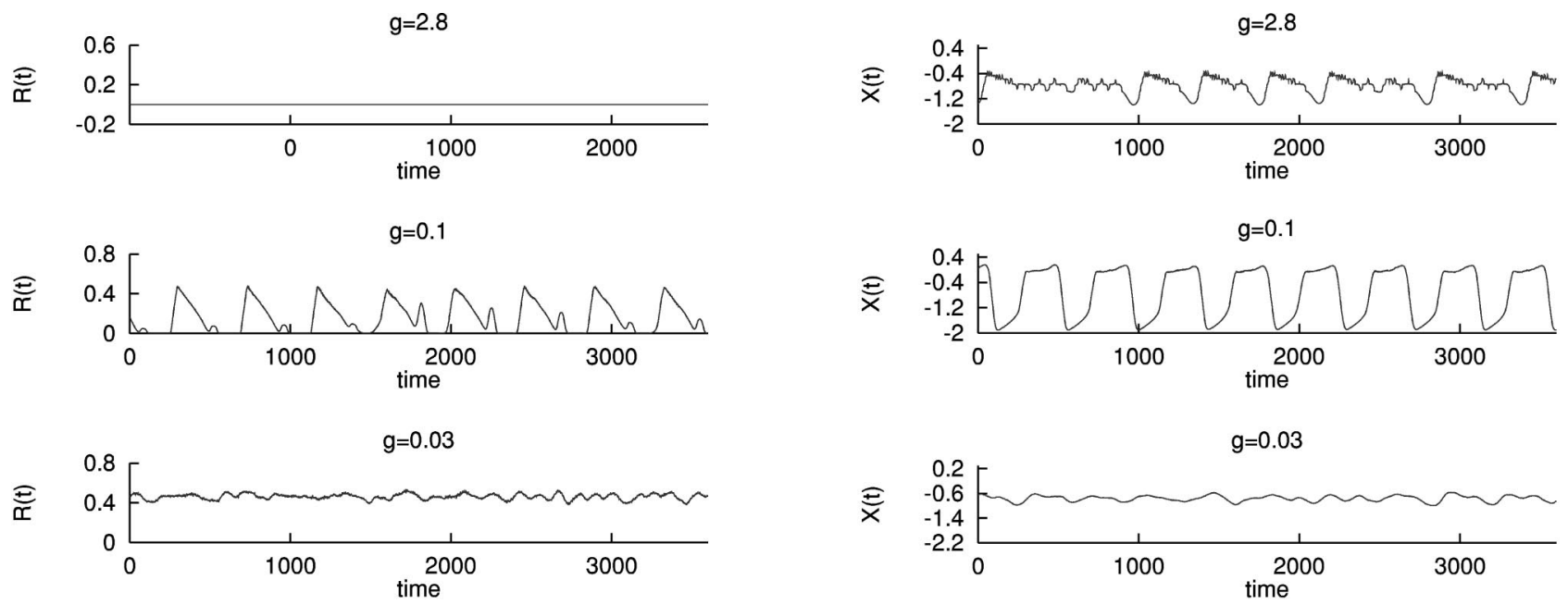

FIG. 1. Left: evolution of $R(t ; g, M)$ as defined in the text for three different coupling strengths $(g)$ in a square network of $10 \times 10 \mathrm{HR}$ elements computed for parameter values $a=3, b=1, c=5, d=1.6, e_{i}=3.281 \pm 0.05, \mu=0.0021$, and $s=4$ (units are dimensionless in this model). Right: average activity $X$ in this network for the same values of $g$.

Ginzburg-Landau model or the Fitzhugh-Nagumo model. To analyze this behavior, we need an equation that describes the average dynamics of a single coarse grain.

We can describe the coarse-grain dynamics using the variables

$$
\begin{gathered}
X(t)=\frac{1}{M} \sum_{i=1}^{M} x_{i}(t)=\left\langle x_{i}\right\rangle_{C G}, \quad Y(t)=\left\langle y_{i}\right\rangle_{C G}, \\
Z(t)=\left\langle z_{i}\right\rangle_{C G},
\end{gathered}
$$

where $M$ is the number of elements in the cluster. An approximate equation for the dynamics of $X, Y, Z$ can be obtained by substituting

$$
\begin{gathered}
x_{i}=X(t)+\xi_{i}(t ; g, M), \quad y_{i}(t)=Y(t)+\eta_{i}(t ; g, M), \\
z_{i}(t)=Z(t)+\zeta_{i}(t ; g, M),
\end{gathered}
$$

in Eqs. (1)-(3), which yields (ignoring higher order terms than $\left.\xi_{i}^{2}\right)$ :

$$
\frac{d X}{d t}=Y+a X^{2}+a r(t ; g, M)-X^{3}-3 X r(t ; g, M)-Z+\epsilon,
$$

$$
\begin{gathered}
\frac{d Y}{d t}=-c X^{2}-Y-[c r(t ; g, M)-b], \\
\frac{1}{\mu} \frac{d Z}{d t}=-Z+s(X+d),
\end{gathered}
$$

where $\epsilon=\left\langle e_{i}\right\rangle_{C G}$. We have taken into account from the definition of $X, Y$, and $Z$ that $\left\langle\xi_{i}(t)\right\rangle_{C G}=\left\langle\eta_{i}(t)\right\rangle_{C G}=\left\langle\zeta_{i}(t)\right\rangle_{C G}$ $=0$ and, consequently, the only function left to be determined is $r(t ; g, M)=\left\langle\xi_{i}^{2}\right\rangle_{C G}$.

In order to describe the slow dynamics, we need to make a reasonable assumption about the nonautonomous term on the right-hand side of Eq. (4). Since $r(t ; g, M)$ varies much more rapidly with time than the slow coarse-grain oscillation, we suppose that the dynamics of a coarse grain depend on the time-averaged value of $r(t ; g, M)$ defined as $R(t ; g, M)=(1 / t) \int_{0}^{t} r\left(t^{\prime}\right) d t^{\prime}$ with $t_{r} \ll t<T$, where $t_{r}$ is the characteristic time scale of the fast pulsation $r(t ; g, M)$ (spiking behavior) and $T$ is the characteristic time scale of $X$. In the system (4)-(6) we now replace $r(t ; g, M)$ with the slow function of time $R(t ; g, M)$ which also depends on the strength of the diffusive coupling between elements and the size of the coarse grain. If our hypothesis is correct, $R$ $\approx$ const $\neq 0$ for small values of $g$ and $R \approx 0$ for large values of the coupling; for moderate values of the coupling, prediction of the behavior of $R$ is not intuitively clear. The computer simulations shown in Fig. 1, however, indicate that for particular moderate values of $g$, the behavior of $R$ becomes periodic. This $g$-dependent behavior of $R$ infers that the averaged dynamics $X$ also will change as the coupling parameter is varied.

We can investigate the appearance of the periodic average behavior using Eqs. (4)-(6) with $r(t ; g, M)$ replaced by $R(t ; g, M)$, the latter being a constant or a periodic function depending on the magnitude of the coupling being considered. Typical phase portraits and the corresponding time series of the full dynamical system for $R$ are presented in Fig. 2. For sufficiently small $g, R$ is nearly constant, taking on values in the range $0.4-0.5$ and only a single stable fixed point appears, one corresponding to steady-state behavior of the cluster. For $R<R_{\text {crit }}\left(g>g_{\text {crit }}\right)$, this fixed point becomes unstable and the limit cycle in the $3 \mathrm{D}$ phase space of the average coarse-grain system undergoes a supercritical and sharp Andronof-Hopf bifurcation to a stable fixed point. Strictly speaking, at the moment of this bifurcation, $R$ becomes a periodic function of time (see Fig. 1). Nevertheless, close to the bifurcation point the influence of this periodicity on the existence of the limit cycle is not important. The direct calculations given in Fig. 2 confirm this supposition.

The dynamical mechanism of the ordering averaged behavior of the coarse grain relies on the synchronization and regularization of the activity of the $M$ units inside the grain. The degree of synchronization of a single neuron with the average activity of the whole grain depends on the strength of the coupling, as one can see on the left in Fig. 3. In the 

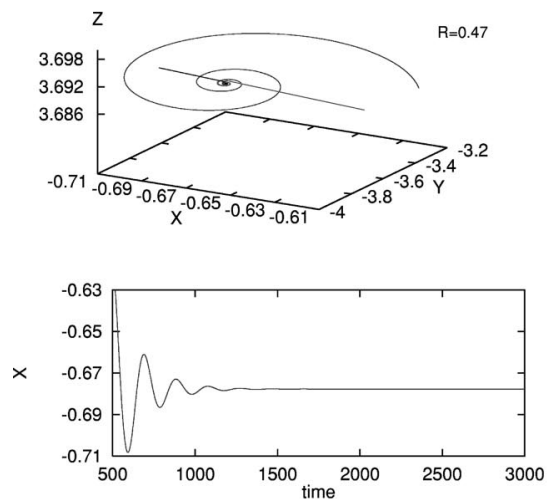
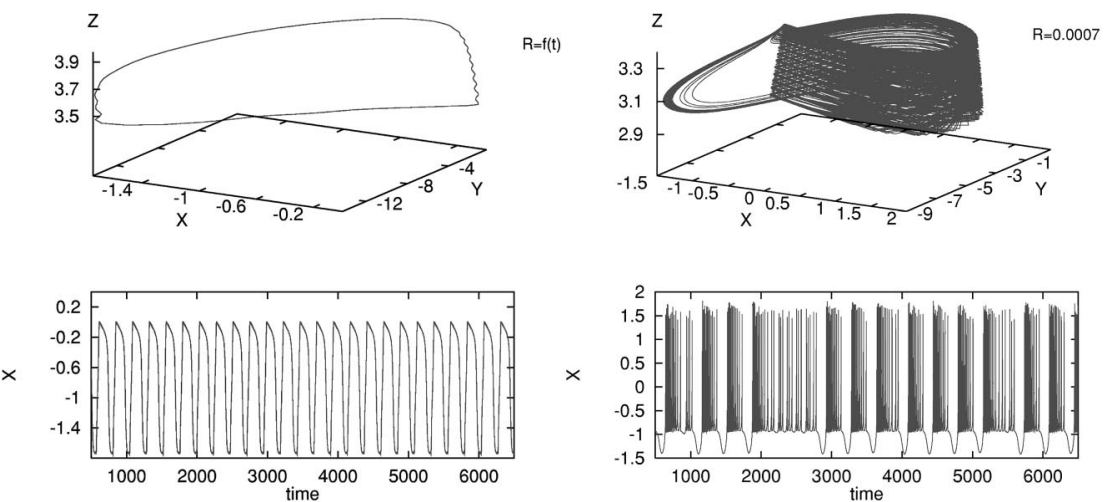

FIG. 2. Phase space portraits (top row) and corresponding $X$ time series (bottom row) of a single coarse-grain element for the parameters given in Fig. 1: $R=0.47$, a stable fixed point; $R=f(t)=\left\{0.25+\sum_{n=1}\left[(-1)^{n-1} / 2 n \pi\right] \sin (2 n \omega t)\right\}\left\{0.5+\sum_{n=1}[2 /(2 n-1) \pi] \sin [(2 n-1) \omega t]\right\}$ $[\omega \approx 0.013$, this periodic function fits the shape of $R(t ; g, M)$ shown in Fig. 1 for $g=0.1]$, a limit cycle; and $R=0.0007$, a strange attractor.

case of regular behavior $(g \approx 0.1)$, the single neuron activity is highly synchronized with the periodic mean field. For $g$ $\approx 0.05$, the synchronization between mean field and individual behavior is absent and one observes spatiotemporal disorder. Thus, for a moderate value of $g$ ( $g \approx 0.1$ using the model parameters described in the figures) the coarse grain behaves as a single element with periodic slow dynamics.

Now we can explain the existence of large $(S \gg 1)$ regular spatiotemporal patterns in discrete diffusive media. First, the existence of such structures is impossible in weakly diffusive media because the local oscillations of neighboring elements are not correlated for small couplings $g$ and the mean field of the coarse grains becomes homogeneous and stable. The direct computation of the Kolmogorov-Sinai entropy presented at the right in Fig. 3 confirms that the level of spatially homogeneous chaos increases as $g \rightarrow g_{0} \ll 1$. Note that this entropy is large for $g=0.1$ since the Lyapunov exponents were calculated from the vector field [13], i.e., without filtering the fast spikes, which are highly chaotic in this regime.

For moderate values of the coupling, the coarse-grain assembly should exhibit regular spatiotemporal patterns. As confirmation of this conjecture, we have checked the behavior of a medium consisting of coarse-grain units with slow periodic behavior. The description of this medium is analogous to that given by the network of HR units wherein $\left(x_{i}, y_{i}, z_{i}\right)$ are replaced by $\left(X_{i}, Y_{i}, Z_{i}\right)$. We are looking for patterns in the coarse grain network that have the same space scale (relative to the size of the lattice) as the pattern in the original HR lattice. Thus, the pattern in the coarse-grain lattice should have identical structure but with a smaller absolute size. Since both patterns (on the original HR lattice and the coarse-grain lattice) have the same time scale, we can say that the speed of front propagation in the HR lattice must be larger than for the coarse-grain lattice. The propagation speed of the front increases together with the value of the diffusion. One concludes, based on this scaling argument, that a coarse-grain pattern with the same relative size as the original may be found only in the case where the coarsegrain lattice coupling $G$ is smaller than the diffusive coupling $g$ in the original HR network (The exact relationship between $g$ and $G$ is beyond the scope of this Rapid Communication and will be reported elsewhere). Verification of this conclusion is given by the sequence of patterns obtained for the square network of coarse-grain units shown in Fig. 4. These patterns (obtained for $G=0.5$ ) given in the bottom row of Fig. 4 have the same topology and are clearly reminiscent of those produced by the original heterogeneous lattice of chaotic HR elements (for $g=1.5$ ) displayed in the top row of Fig. 4. Periodic boundary conditions were applied to both $\mathrm{HR}$ and coarse-grain networks in all the simulations described in this paper. The topology of the patterns was not affected when we used fixed boundary conditions. We have also observed the same behavior in hexagonal lattices (both
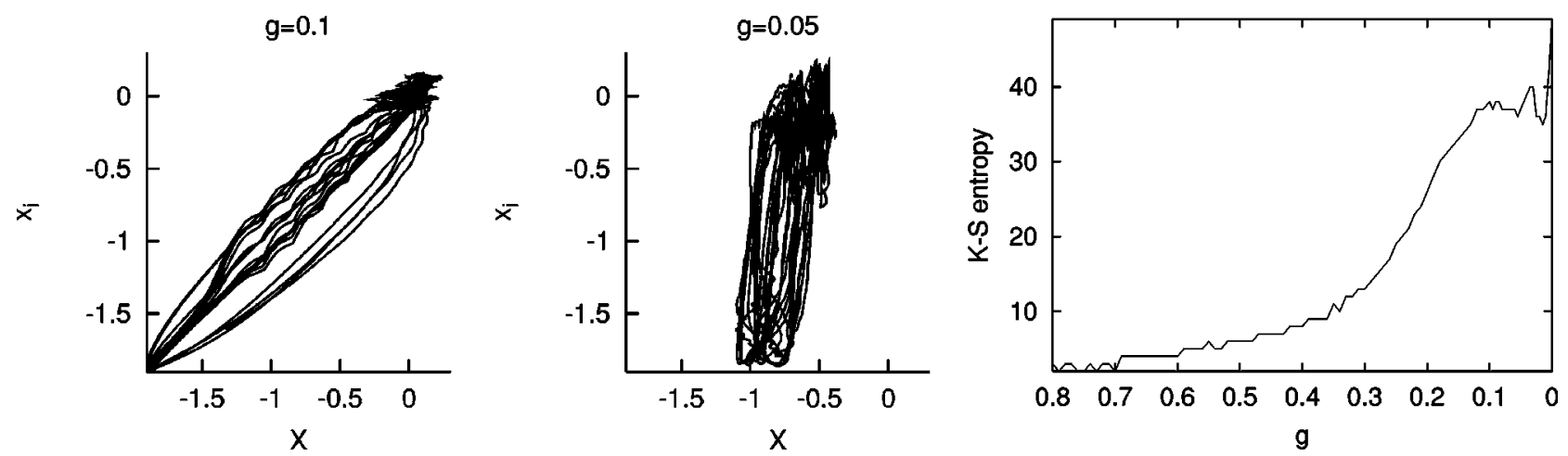

FIG. 3. Left: activity of a single HR unit $x_{i}$ vs the average activity $X$ (defined in the text) for two values of the coupling $g$. Right: Kolmogorov-Sinai entropy (sum of the positive Lyapunov exponents obtained from the vector field) as a function of the coupling strength $g$ in a network of $7 \times 7$ HR elements. 

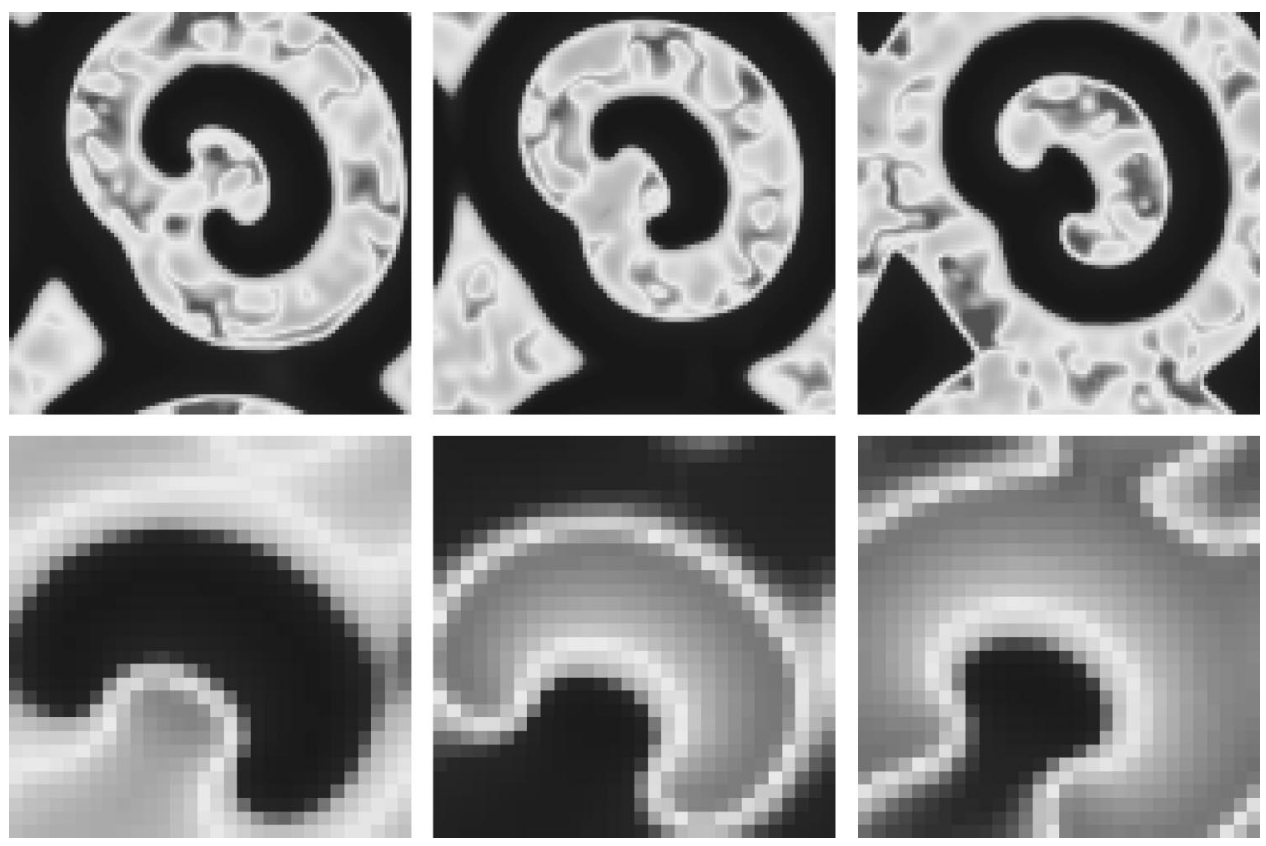

FIG. 4. Top row: evolution of a periodic spatiotemporal pattern observed in a network of $100 \times 100$ HR elements; parameter values are those specified in Fig. 1, with $g=1.5$. Bottom row: Periodic spatiotemporal patterns observed in a network of $30 \times 30$ coarse-grain elements computed for $R=0.23$ and $G=0.5$ (the rest of the parameters have the same values used in the HR lattice). The value of $R$ is close to the bifurcation point and the individual coarse-grain dynamics is periodic.

coarse grains and HR networks). In this case, the strength of the coupling has to be reduced to take into account the larger number (six) of nearest neighbors.

We conclude that the formation of large-scale coherent structures in nonequilibrium media consisting of discrete HR chaotic elements with fast and slow oscillations exhibits two key features. The first is the regularization phenomena inside clusters of chaotic elements, i.e., the coarse grains. This regularization of the behavior is the result of the action of the averaged activity of fast pulsations in the slow coarse-grain dynamics. The second feature is the instability of the homo- geneous oscillation modes in a media considered to be a coarse-grain lattice. Also, it is important to remind the reader that the coarse grains are a temporal assembly of neurons whose relaxation time is smaller than the relaxation time of the coherent structures.

We thank H.D.I. Abarbanel, V. Afraimovich, and Y. Sinai for useful discussions. M.I.R. and R.H. acknowledge support from the U.S. DOE and the NSF. J.J.T. and P.V. acknowledge support from Universidad de Granada and M.E.C., respectively.
[1] D. J. Tritton, Physical Fluid Dynamics (Clarendon, Oxford, 1988).

[2] J. C. Prechtl, L. B. Cohen, B. Pesaran, P. P. Mitra, and D. Kleinfeld, Proc. Natl. Acad. Sci. USA 94, 7621 (1997).

[3] I. A. Shevelev, E. N. Tsicalov, A. M. Gorbach, K. P. Budko, and G. A. Sharaev. J. Neurosci. Methods 46, 49 (1992).

[4] B. J. Gluckman, P. Marcq, J. Bridger, and J. P. Gollub, Phys. Rev. Lett. 71, 2034 (1993).

[5] D. P. Lathrop, J. Fineberg, and H. L. Swinney, Phys. Rev. Lett. 68, 1515 (1992).

[6] L. Ning, Y. Hu, R. E. Ecke, and G. Ahlers, Phys. Rev. Lett. 71, 2216 (1993).
[7] R. Huerta, M. Bazhenov, and M. I. Rabinovich, Europhys. Lett. 43, 719 (1998).

[8] J. L. Hindmarsh and R. M. Rose, Proc. R. Soc. London, Ser. B 221, 87 (1984).

[9] X.-J. Wang, Physica D 6, 263 (1993).

[10] R. Huerta, M. I. Rabinovich, H. D. I. Abarbanel, and M. Bazhenov, Phys. Rev. E 55, R2108 (1997).

[11] V. S. Afraimovich, N. N. Verichev, and M. I. Rabinovich, Inv. VUZ Radiofiz. 29, 795 (1986).

[12] L. M. Pecora and T. L. Carroll. Phys. Rev. Lett. 64, 821 (1990).

[13] T. S. Parker and L. O. Chua, Practical Numerical Algorithms for Chaotic Systems (Springer, New York, 1989). 